\title{
Efficient Iris and Eyelids Detection from Facial Sketch Images
}

\author{
Tan Boonchuan*, Samsul Setumin*,+, Abduljalil Radman* and Shahrel Azmin Suandi* \\ ${ }^{*}$ Intelligent Biometric Group, School of Electrical and Electronics Engineering, Universiti Sains Malaysia, \\ Engineering Campus, 14300 Nibong Tebal, Pulau Pinang, Malaysia. \\ + Faculty of Electrical Engineering, Universiti Teknologi MARA Pulau Pinang, \\ Jalan Permatang Pauh, 13500 Permatang Pauh, Pulau Pinang, Malaysia.
}

Received 09th Jan 2017; accepted 24th Jul 2018

\begin{abstract}
In this paper, we propose a simple yet effective technique for an automatic iris and eyelids detection method for facial sketch images. Our system uses Circular Hough Transformation (CHT) algorithm for iris localization process and a low level grayscale analysis for eyelids contour segmentation procedure. We limit the input face for the system to facial sketch photos with frontal pose, illumination invariant, neutral expression and without occlusions. CUHK and IIIT-D sketch databases are used to acquire the experimental results. As to validate the proposed algorithm, experiments on ground truth for iris and eyelids segmentation, which are prepared at our lab, is conducted. The iris segmentation from the proposed method gives the best accuracy of 92.93 and 86.71 based on F-measure evaluation for IIIT-D and CUHK, respectively. For eyelids segmentation, on the other hand, the proposed algorithm achieves an average of 4 standard deviation which indicates the closeness of proposed method to ground truth.
\end{abstract}

Key Words: iris, eyelids, circular hough transform, sketch images

\section{Introduction}

In law enforcement, the use of facial sketch to match and narrow down the criminal suspect is considered common. It was, however, very time consuming as the process was done manually. Automatic retrieval system is capable to significantly reduce the searching time but it is quite a challenging task as the sketch and digital images come from different modalities [1,2,3]. Different modalities introduce a substantial gap between the sketch and real photo. The gap can be reduced by performing synthesizing process on either the sketch or photo to make up a pseudo-sketch/photo. When both images are in the same domain, most of the existing recognition approaches for real face photo can simply be used to recognize the facial sketch [2]. The subsequent works have already boosted the state-of-the-art performance in face sketch recognition $[4,5,6,7]$.

Recently, forensic sketch recognition has received much attention as the matching performance of existing methods for this type of sketches remains significantly low [8]. Forensic sketches are the sketch drawn by a professional forensic artist based on descriptions given by an eyewitness or the victim. Due to a symbolic

Correspondence to: <shahrel@usm.my>

Recommended for acceptance by Luiz Goncalves

https://doi.org/10.5565/rev/elcvia.1044

ELCVIA ISSN: 1577-5097

Published by Computer Vision Center / Universitat Autonoma de Barcelona, Barcelona, Spain 
representation of a forensic sketch to its corresponding real photo, researchers like [9] and [10] have started to take a different approach to represent the sketch and photo that is invariant to modality difference. This allows the sketch-photo comparison to be made between two consistent features representation. Feature extraction based on strokes composition analysis [11] for each facial components (e.g., eyes, nose and mouth) is an alternative algorithmic solution for matching face sketch to photos. It is done by obtaining descriptive stroke characteristics from the extracted facial component to represent the shape of object of interest. This methods will enable a system to describe the stroke composition on every facial component in detail manner for both pseudo-sketch and real forensic sketch.

In order to extract the salient stroke features of each face component from both the sketch and photograph, component detection play an important role towards achieving this goal. Eyes are among the component of interest for face component-based sketch-photo matching. The eyes feature can be extracted from the pattern of eyes subcomponent, i.e., iris and eyelids. In this paper, an efficient iris and eyelids detection technique from facial sketch images is presented. The remaining of this paper is organized as follows: Section 3 presents the proposed method to detect the iris and eyelids. The experimental results and conclusion are given in Section 4 and Section 5, respectively.

\section{Theoretical Background}

Human face is one of the unique body feature that can be used to differentiate human. Therefore, automatic face recognition has been applied, studied and researched for more than 30 years in many field that include personal ID, identification, human-centric computing, mug-shot searching, surveillance systems and law enforcement [24]. It is widely used because of the fact that everyone has different shape and description of the facial components such as eyes, eyebrows, nose, ears, and face outline. The shape is more obvious for facial sketches as the forensic artist normally emphasizes the shape and edge using different pencil strokes. Note that facial sketch has useful application in criminal investigation that is to narrow down the potential suspect. The sketch is called forensic sketch and it is drawn by forensic artist using pencil. Hence, the sketch is rendered from different modality as compared to its corresponding photo. To match this kind of images, the robust feature is required in order to extract the salient information of the sketched images.

Face detection is the first stage of the face recognition system that is used to extract region of the images that contain faces. The popular method to perform this detection is proposed by Viola and Jones [15]. Next, the facial components are extracted within this region. The fact that strokes composition analysis [11] for each facial components (e.g., eyes, nose and mouth) is an alternative algorithmic solution for matching face sketch to photos, therefore, obtaining descriptive stroke characteristics from the extracted facial component to represent the shape is crucial. This methods will enable a system to describe the stroke composition on every facial component in details. In order to extract the salient stroke features from each facial component, the component detection is mandatory. Eyes are among the component of interest for face component-based sketch-to-photo matching. Then, the eyes feature can be extracted from the pattern of eyes subcomponent, i.e., iris and eyelids. Here, the baseline method for iris segmentation is the method proposed by Zhao and Kumar [23]. According to the study in [25], the taxonomy of the eye detection methodologies can be categorized as shape-based approaches, feature-based approaches, appearance-based approaches, and hybrid approaches.

Shape-based methods can be divided into fixed shape and deformable shape. The deformable templates parameter are changeable in term of their size and others parameter value as to match themselves to the data. The final values of these parameter are used to describe the features. The advantages of the deformable template are this method can still work regardless of variation in scale, tilt, rotation of head, and lighting condition. However, there are some limitation, which include the computational demanding, require high contrast images and unable to handle face with pose variation and eye occlusions well. Hansen and Ji [25] divided the shapebased approaches into simple elliptical shape-models and complex shape models. The complex shape model example is deformable eye template method. Simple ellipse models consists of voting-based methods and model fitting methods. Voting methods works such that it select features that support a given hypothesis through 
a voting or accumulation process, while model fitting approaches fit selected features to the model for example ellipse.

Feature-based methods identified a set of distinctive features around the eye. The limbus, pupils, and cornea reflections are common features used for eye localization. Feature-based approaches tend to identify informative local features of the eye and face that are less sensitive to variations in illumination and viewpoint. Feng and Yuen [26] described the eye model which consists of six landmarks. The eye model consists of three components, namely iris, upper eyelid and lower eyelid. Furthermore, Feng and Yuen [27] proposed variance projection function (VPF) to determine the eye position precisely. VPF used the intensities variation within the eye region to estimate the position and size of the iris or the position of the eyelids. VPF is able to detect eye position which is robust to orientation and scale invariant. However, the false results are obtained if the eye are covered partially by the hair and the false eyebrows detection as eye. Pupil and iris are reliable features for eye detection. This is because pupil and iris have darker pixel intensity than surrounding region. Stiefelhagen and Waibel [28] introduced iterative threshold algorithm to detect the pupil and iris by searching two dark regions that satisfy certain anthropometric constraints using a skin-color model. However, this technique yield bad performance by the presence of other dark regions such as eyebrows and shadows. Moreover, this method is limited to the grayscale images like sketch images. The feature-based methods provide good robustness during illumination changes during eye detection. However, the performance of this technique is affected by the imperfect eye region detected such that the eye region enclosed by hairs, closed eye, wrong eye region detected and it is high computational complexity.

The appearance-based methods are also known as image template or holistic methods. The appearancebased methods detect and track eyes directly, based on the photometric appearance as characterized by the color distribution or filter responses of the eye and its surroundings. Image template-based methods unable to counter the problem with scale and rotational changes during eye detection. Holistic approaches applied statistical techniques to analyze the intensity distribution of the entire object appearance. The appearance-based methods usually collect a large amount of training data representing the eyes of different subjects, under different face orientations and different illumination conditions, but the underlying models are essentially independent of the object class. A classifier or regression model can be constructed through the model of pixel variations. Huang et al. [29] and Jiao and He [30] detect and track the eye region using support vector machine (SVM) and proved that the polynomials with second degree kernels yields best generalization performance. Moreover, Hillman et al. [31] introduced the Eigen analysis (PCA on image vectors) of image templates to model variations in data training of the eye. PCA helps to improve detection efficiency and accuracy of eye detection. Moreover, Fasel et al. [32] applied the Haar features that have similar approaches with the Viola and Jones detection algorithm [15] to detect eye region using Adaboost method. Although Haar features are computational efficiency and easy to compute, they have limited discriminating efficiency.

Hybrid-based method used the combination of shape and appearance within a single system in eye detection through part-based approaches. Kuo and Hannah [33] proposed a part-based model employing a prior shape model consisting of several subcomponents. Firstly, the eye region is detected using threshold and binary search. After that, the eye region is divided into several parts which included the whole eye region, two regions representing sclera and the iris. The irises and the eyelids are then modeled by circles and parabolas that have predetermined parameters and intensity distribution. In addition to that, Hansen et al. [34] suggested shape and appearance model combination through Active Apperance Model (AAM). In these models, both shape and appearance are combined into one generative model. The model can then be fitted to the image by changing the parameters according to a learned deformation model.

\section{Proposed Method}

In this paper, we propose a technique for iris localization and eyelids contour detection prior to the segmentation process. The technique includes image acquisition, image pre-processing, face detection, eye region extraction, iris detection and eyelids detection. Figure 1 shows the overall process flowchart of the proposed method. The 
detection process starts with the image acquisition from two different sketch databases, that are CUHK [12, 13] and IIIT-D [14] databases. The use of two different database in this experiment is to test the robustness of the feature extraction system. Next, the image is resized before the face detection procedure is performed. After the face region is obtained, the eye pair region is detected. Then, the right eye and left eye are segmented separately from the big eye pair to become a single eye region by applying geometrical method. After the single eyes for each face are successfully acquired, iris from both eyes are detected separately. From each single eye region, the area of eyelids for both eyes are determined from iris information. Some image pre-processing and image morphological processes are also applied to the eye image to estimate the area of the eyelids. We select the Region of Interest (ROI) based on Equation (1) and (2).

$$
\begin{gathered}
R O I_{\text {left }}=\left[x_{l}, y_{l}, \text { width }_{l}, \text { height }_{l}\right]=\left[x^{\prime}, y^{\prime},\left(x_{c}-\beta\right)-x^{\prime}, 2 r\right] \\
R O I_{\text {right }}=\left[x_{r}, y_{r}, \text { width }_{r}, \text { height }_{r}\right]=\left[x_{c}+\beta, y^{\prime}, x^{\prime \prime}-\left(x_{c}+\beta\right), 2 r\right]
\end{gathered}
$$

Here, $\beta=\alpha r$ in which $r$ is the radius of detected iris and $\alpha$ is the coefficient. We set $\alpha=1.2$ in our proposed method. $x^{\prime}$ and $x^{\prime \prime}$ are the first and last $x$-coordinate of the detected single eye region, respectively. $x_{c}$ is $x$-coordinate of iris center. $y^{\prime}$ is the $y$-coordinate at upper eyelids crossing point.

This is to detect the eyelids contour points and to eliminate the unwanted region of the image so that the accuracy of the algorithm can be increased. The corner points for both eyes are detected by observing the pixel intensity of the image. In addition to that, the upperlid and the lowerlid of each eyes are formed by using least square error curve fitting technique after getting the points of the eyelid contour from the previous step. To determine the accuracy of the iris location as well as eyelids detection, the ground truth for the eye images are created by manually determining the real position of the subject. The Euclidian distance, absolute mean error, and F-measure are calculated between experimental results and the ground truth to validate the proposed method.

\subsection{Pre-Processing}

The first step for iris and eyelids detection is to acquire the probe image. In our experiment, the probe images are retrieved from CUHK testing sketch and IIIT-D sketch databases. There are a total of 172 images from CUHK database (100 images) and IIIT-D database (72 images). The loaded image is then geometrically normalized such that the position of the eyes is located at the centre of the image and horizontally alligned. It is then scaled to $414 \times 582$ dimensions (i.e. based on empirical data) by employing bicubic interpolation.

Subsequently, face region is extracted by applying face detection algorithm. This is executed before eyes, iris and eyelids segmentation took place. It begins with face region localization and followed by a cropping process of the region of interest so that only related pixels portion is fed into the subsequence processes. This is to enhance the performance and accuracy of the feature extraction algorithm. We adopt Viola and Jones [15] method to detect the face region. In order to make the detection algorithm targets the correct face candidate and the fact that we are only interested to extract a single face region (e.g. multiface detection error), only the largest region of interest (i.e. face candidate) is segmented as the target face. This assumption is valid due to the input face photos for this system is generated in such a way that it is in frontal pose with close up face.

After face region from the probe sketch is segmented, the eye extraction algorithm is employed. The same approach for face extraction is utilized here. The extraction process can be divided into two stages, which are big eye pair detection and single eye segmentation. Firstly, the eye pairs position is localized. In this process, instead of using the entire face region, we propose to use only the 60 percent from the top of the face region to detect the eye pairs. Once detected, the geometric model [16] can be used to separate the region of right eye and left eye. We apply the geometrical method to extract the right eye and left eye regions due to the fact that both eyes have symmetrical geometrical properties. 


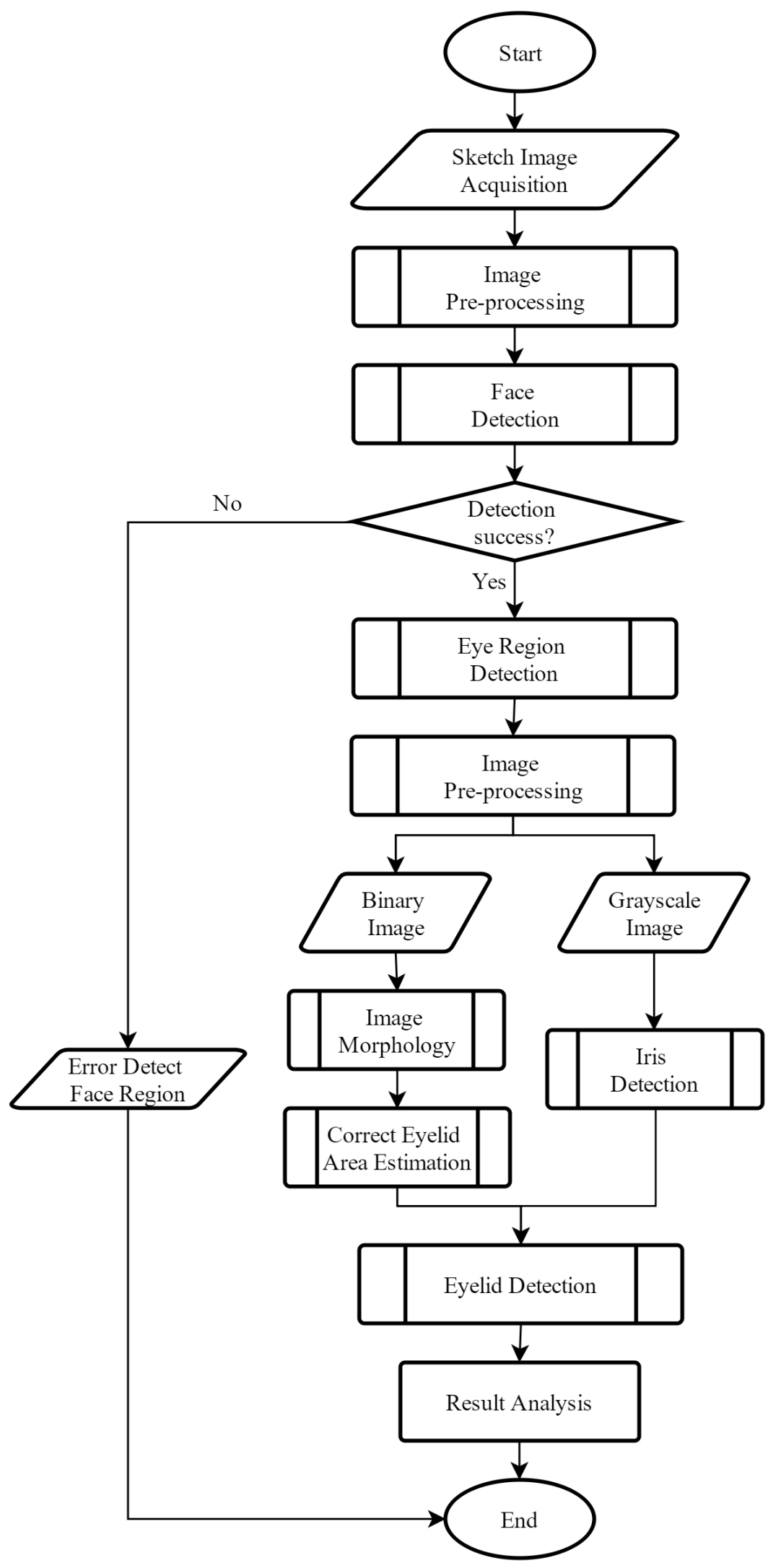

Figure 1: Overall process flowchart of the proposed method 


\subsection{Iris Detection}

In order to detect the iris position from the eye images, Circular Hough Transform (CHT) method is used $[17,18]$. This is because the human eye iris is in the circular form and hence the black circle from the eye image is considered to be the eye iris $[19,20]$. Although this approach is robust to noisy images but we insist to apply a Gaussian filter to reduce any noise and to avoid false circle detection. Other than that, we perform simple pre-processing procedure to enhance the detection performance. This is done by converting the input image into a binary image by using a thresholding operation. It is then followed by a morphological opening and closing to remove background noise and small holes, respectively.

The CHT algorithm $[17,18]$ works by three fundamental steps that begin with computational of an accumulator matrix, followed by estimating the center point and eventually estimating the radius of detected circle. In the parameter space, to find the crossing point, we first divide the parameter space into a number of bins and introduce an accumulator matrix according to the grid. The element in the accumulator matrix is the recorded number of potential circle in the parameter space. It counts potential circle in the parameter space that passing through the corresponding grid cell in the parameter space. This number will keep increasing as it is called "voting" process. After voting, the circle centers are estimated by detecting the maximum number of vote in the accumulator matrix. Hence the radius of the circle is ready to be estimated. To improve the detection performance based on our empirical data, we assist the algorithm by specifying the radius ranges between 5 to 30.

\subsection{Eyelids Detection}

After acquiring the iris position, the eyelid contour points are detected. Firstly, the eyelid areas are estimated by measuring the vertical height of the upper eyelid and lower eyelid. Then the region of interest of the eyelid contour points are selected to eliminate unwanted region from the image. Finally, the eyelids' points are detected followed by the eyelid contour formation by using polynomial curve fitting [21]. Vertical difference between upper eyelid and lower eyelid is determined by using the center point of the detected iris. Prior to this, some image pre-processing procedures are conducted, which include image binarization and holes filling morphology. After the image is converted into binary image, the iris is dark in pixel intensity, which is represented as 0 , while the background pixels are bright, which is represented as 1 in pixel intensity. To determine the height of the eyes, the pixel intensity from the center of the iris is scanned in descending order from $y$-axis pixel-by-pixel. The upper position of the eye is predicted if there is a change in pixel intensity from black pixel to white pixel.

Before the eyelid points are detected, the region of interest is set to remove unwanted area from the image and hence, reduce the percentage of the noise which occurs during points detection stage. After the points of the eyelids contour have been found, the eye corners are determined. The eye corners are important in order to separate the eyelids into upper eyelid region and lower eyelid region. Once the points of eyelids contour are acquired, the 2 eye corners points are predicted such that the left eye corner has the lowest value of column value whereas the right eye corner has the largest value of the column value. To determine the lower eyelid or upper eyelid contour points, firstly, the points are separated into 2 regions. The points which have the column value that are lower than the column position of the eye iris are set as the left region. On the other hand, the points which have the column position higher than the column position of the iris are declared as the right region. In other words, the points are separated into 2 regions, which are left region and right region. This is because the two eye corners might have different row value position. Therefore, it is essential to divide the eye contour part into 2 regions for accurate result of the eyelid formation stage. After that, for each region, the points which have the row value that are lower than the eye corner point located in the same region are set as the upper eyelid contour, whereas the points which have the row value higher than the eye corner in the same region are declared as the lower eyelid contour.

$$
y=p(x)=P_{1} x^{n}+P_{2} x^{n-1}+\ldots+P_{n} x+P_{n+1}
$$


In this paper we propose the use of polynomial curve fitting [21] as defined in Equation (3) for the eyelid formation. Additionally the least square error technique is also employed to assist the curve fitting process. When Equation (3) is computed, it will return the coefficients for a polynomial $p(x)$ of degree $n$ that is a best fit (i.e. in a least-squares sense) for the data in $y$. From Equation (3), $(x, y)$ indicates a set of points' coordinates and the coefficient in $p(x)$ includes $\left(P_{1}, P_{2}, P_{3}, \ldots, P_{n+1}\right)$. The coefficients in $p(x)$ are in descending powers and the length of $p(x)$ is $n+1$. After acquire the coefficients in $p$, the polynomial evaluation is then conducted to find out the value of $y$ that fits the polynomial curve (Equation (3)) with respect to the value of $x$.

\section{Experimental Results}

To determine the performance of proposed method, we have conducted two different evaluations; which are qualitative and quantitative evaluation. All have been conducted on a PC equipped with Intel i5 CPU $2.5 \mathrm{GHz}$ 6GB RAM on Windows 8 OS. On one hand, qualitative evaluation in our experiments is related to the performance evaluation that is not easily measurable by the data and this evaluation has been carried out through Human Visual System (HVS). On the other hand, our quantitative evaluation refers to the data interpretation that relies on statistical methods and mathematical evaluation. For that, quantitative evaluation is conducted by comparing the experimental results with the ground truth value.

\subsection{Qualitative Evaluation Results}

Qualitative evaluation is implemented to evaluate face detection and eye detection system's accuracy. Table 1 shows the results of Viola and Jones [15] and proposed method for face extraction. Figure 2 shows some false face detection results using [15]. There are multiple regions of interest (i.e. blue rectangle) detected and this will affect the result in the next processing stage. Therefore, false regions need to be eliminated to acquire true face region from the sketch image. Figure 3 shows examples of true face detection results from both IIIT-D and CUHK database.

Table 1: Face successful detection rate

\begin{tabular}{|c|c|c|}
\hline \multirow{2}{*}{ Database } & \multicolumn{2}{|c|}{ Face Detection Result (\%) } \\
\cline { 2 - 3 } & Viola and Jones [15] & Proposed Method \\
\hline IIIT-D Sketch (72 images) & 95.83 & 98.61 \\
\hline CUHK testing sketch (100 images) & 100.00 & 100.00 \\
\hline
\end{tabular}

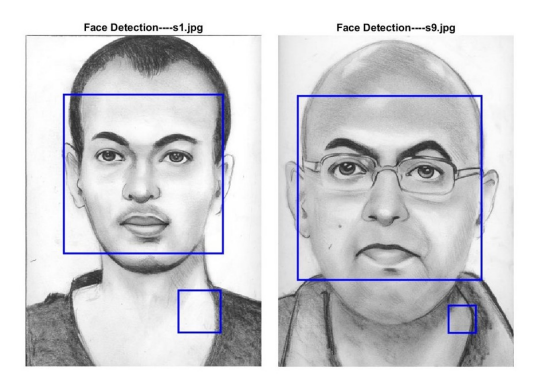

Figure 2: False face detection by using Viola and Jones [15] face detection method.

Table 2 shows the results of the eye pair detection method. From this table, it shows that the proposed method is able to detect correct eye pair region more accurately as compared to [15]. The proposed method (i.e. geometrical method) successfully detects 71 eye pair regions from IIIT-D database compared to only 63 


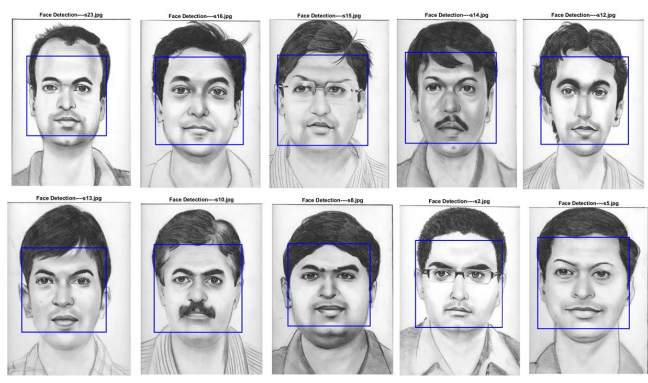

(a) IIIT-D sketch database
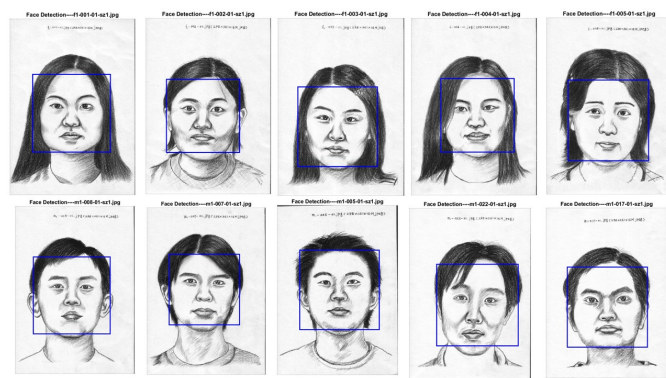

(b) CUHK sketch database

Figure 3: True face detection results from IIIT-D and CUHK database in (a) and (b), respectively.

successful eye pair detections by using [15]. This false detection is due to nostril or eyebrow region mistakenly predicted as the eye pair region. This demonstrates that the detection algorithm perceives shape and appearance of nostril and eyebrow as an eye. To address this problem, we propose to merely utilize upper face region eye region detection. As a result, false eye detection is reduced significantly.

Table 2: Eye pair successful detection rate

\begin{tabular}{|c|c|c|}
\hline \multirow{2}{*}{ Database } & \multicolumn{2}{|c|}{ Eye Pair Detection Result (\%) } \\
\cline { 2 - 3 } & Viola and Jones [15] & $\begin{array}{c}\text { Proposed Method } \\
\text { (Geometrical Method) }\end{array}$ \\
\hline IIIT-D Sketch (72 images) & 87.50 & 98.61 \\
\hline CUHK testing sketch (100 images) & 96.00 & 99.00 \\
\hline
\end{tabular}

\subsection{Quantitative Evaluation Results}

Quantitative evaluation is carried out to evaluate the iris and eyelids segmentation system's accuracy. The evaluation refers to the data interpretation that relies on statistical methods and mathematical evaluation. In addition to that, the evaluation results shown in the subsection below are based on evaluations that are conducted by comparing the experimental results with the ground truth value. However, due to unavailability of iris and eyelids ground truth data from both original database, all ground truth have been generated at our lab.

\subsubsection{Iris Segmentation Evaluation}

In order to show the accuracy of our iris segmentation system quantitatively, the results are compared with the ground truth results based on the detected iris center position and radius value. Intersection methodology between the ground truth radius mask and the proposed method mask is applied [22]. The utilization of the precision, recall and F-measure determines the eye iris segmentation performance. These precision, recall and F-measure are defined as $\mathcal{P}, \mathcal{R}$, and $\mathcal{F}$ in Equation (4), Equation (5) and Equation (6), respectively. The process is illustrated in Figure 4.

$$
\begin{aligned}
& \mathcal{P}=\frac{t p}{t p+f p} \\
& \mathcal{R}=\frac{t p}{t p+f n} \\
& \mathcal{F}=\frac{2 \mathcal{R} \mathcal{P}}{\mathcal{R}+\mathcal{P}}
\end{aligned}
$$




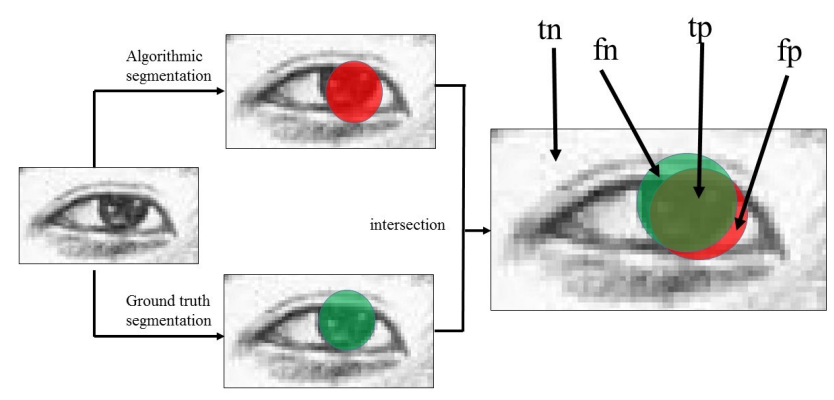

Figure 4: Intersection method for eye iris segmentation evaluation. Red color demonstrates the region detected by proposed method while green color illustrates the ground truth.

Precision $(\mathcal{P})$ can be defined as the percentage of retrieved iris pixels which are correct. In addition, Recall $(\mathcal{R})$ defined as the percentage of the iris pixels in ground truth which are correctly retrieved. F-measure is the harmonic mean of the precision and recall, which is the combination for the precision and recall since the target is to optimize both scores, which are precision and recall.

From Figure 4, the intersection mask can be divided into 4 groups, true positive $(t p)$, true negative $(t n)$, false positive $(f p)$ and false negative $(f n)$. Table 3 indicates the descriptions of these groups, while Table 4 shows the accuracy of the iris segmentation in terms of precision, recall and F-measure. From Table 4, IIIT-D database has better accuracy in the iris segmentation procedure. This is because IIIT-D database has higher F-measure value than CUHK database, which are 92.93 and 86.71 , respectively.

Table 3: Descriptions of the intersection mask in Figure 4

\begin{tabular}{|c|c|}
\hline Group & Description \\
\hline True Positive $(t p)$ & The number of iris pixels which were correctly marked \\
\hline True Negative $(t n)$ & The number of unmarked non-iris pixel \\
\hline False Positive $(f p)$ & The number of non-iris pixels which were marked \\
\hline False Negative $(f n)$ & The number of unmarked iris pixels \\
\hline
\end{tabular}

Table 4: Accuracy evaluation of the iris segmentation in terms of precision, recall and F-measure

\begin{tabular}{|c|c|c|c|c|c|c|}
\hline \multirow{2}{*}{ Database } & \multicolumn{2}{|c|}{ Precision } & \multicolumn{2}{c|}{ Recall } & \multicolumn{2}{c|}{ F-Measure } \\
\cline { 2 - 7 } & mean & s. deviation $( \pm)$ & mean & s. deviation $( \pm)$ & mean & s. deviation $( \pm)$ \\
\hline IIIT-D & 93.85 & 13.58 & 92.65 & 12.38 & 92.93 & 12.20 \\
\hline CUHK & 95.64 & 10.25 & 80.48 & 14.66 & 86.71 & 11.76 \\
\hline
\end{tabular}

\subsubsection{Eyelids Segmentation Evaluation}

Figure 5 shows the eyelids contour detection results based on Human Visual System (HVS). From the figure, false detection of eyelids are caused by inaccurate identification of the eye corners point, therefore, the eyelids areas are deviated from the actual contour shape.

Quantitatively, the accuracy of the eyelid contour segmentation is evaluated by computing the Euclidian distance from eyelid contours points to the ground truth. One eye consists of 2 eyelids, which are upper eyelid and lower eyelid. Hence, for computation purpose, the 2 eyelids are represented by 100 points which lies on the eyelid contour. Then, the Euclidian distance of those points are computed.

Table 5 shows the eyelid contour segmentation in absolute mean error and its' standard deviation. From the table, IIIT-D database has higher standard deviation value than CUHK database which are 6.2474 and 3.9783, 


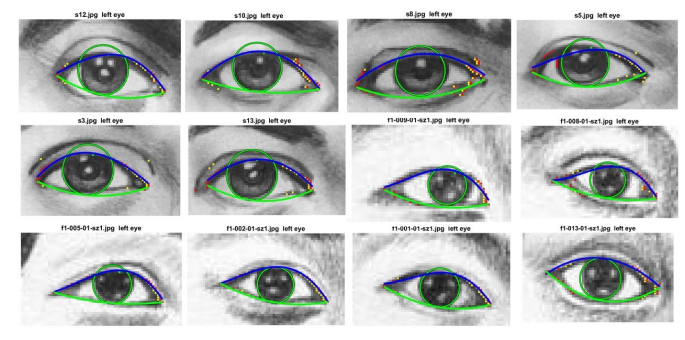

(a) True eyelids detection

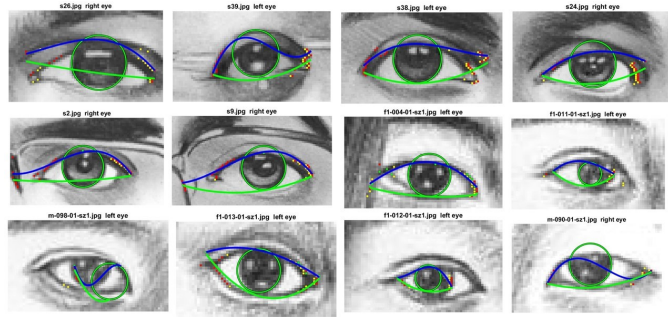

(b) False eyelids detection

Figure 5: Eyelids detection results evaluation based on HVS.

respectively. This shows that IIIT-D database have bigger error on eyelid contour formation when compared to CUHK database.

Table 5: Absolute Mean Error and Standard Deviation of the eyelid contour segmentation evaluation

\begin{tabular}{|c|c|c|}
\hline Database & Absolute Mean Error & Standard Deviation $( \pm)$ \\
\hline IIIT-D & 4.1205 & 6.2474 \\
\hline CUHK & 2.2172 & 3.9783 \\
\hline
\end{tabular}

\subsection{Comparison between Proposed Method and Existing Method}

In this section, the proposed method for iris and eyelids segmentation is compared with the method from Zhao and Kumar [23]. Table 6 shows the F-measure of the iris segmentation results from both approaches. From the table, the proposed method has better F-measure value of 92.93 in iris segmentation whereas [23] only has obtained 84.98. This demonstrates that the proposed method has better performance than [23] in iris segmentation.

Table 6: F-measure of the iris segmentation evaluation from different methods

\begin{tabular}{|c|c|c|c|}
\hline \multirow{2}{*}{ Method } & \multirow{2}{*}{ Database } & \multicolumn{2}{|c|}{ F-measure } \\
\cline { 3 - 4 } & & Mean & $\begin{array}{c}\text { Standard } \\
\text { Deviation }( \pm)\end{array}$ \\
\hline \multirow{2}{*}{ Proposed Method } & IIIT-D & 92.93 & 12.20 \\
\cline { 2 - 4 } & CUHK & 86.71 & 11.76 \\
\hline \multirow{2}{*}{ Zhao and Kumar [23] } & IIIT-D & 84.98 & 20.10 \\
\cline { 2 - 4 } & CUHK & 83.00 & 14.07 \\
\hline
\end{tabular}

For eyelid segmentation, the proposed method has more precise and accurate eyelids contour formation when compared to [23]. Figure 6 shows the comparison of eyelids contour formation by proposed method and [23]. The first row illustrates eyelid contour formation results from [23] whereas the second row illustrates the eyelids segmentation from proposed method. From the figure, the proposed method has better eyelid segmentation based on HVS. [23] only provides the upperlid contour formation. Eye corners are not computed, which therefore, unable it to form perfect eyelids region.

\section{Conclusion}

The performance of proposed method has been evaluated qualitatively and quantitatively by using different sketch databases. The iris segmentation from proposed method gives the average accuracy of 92.93 and 86.71 


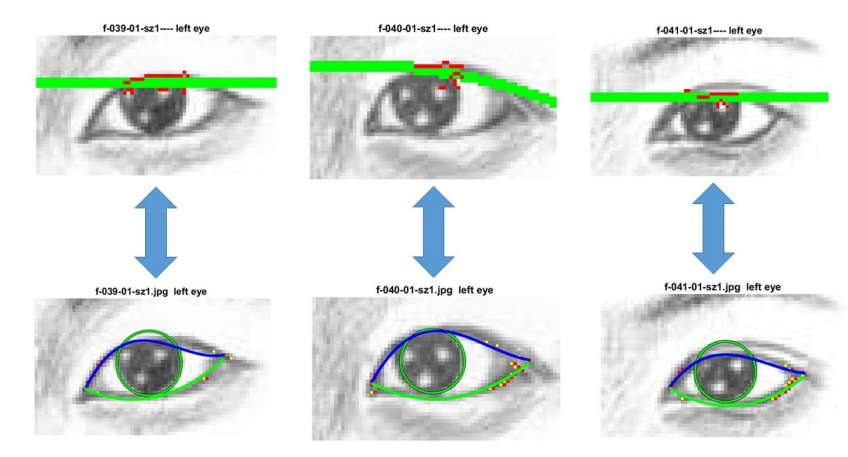

Figure 6: Eyelid contour formation using [23] (top row) and proposed method (bottom row).

based on F-measure evaluation from IIIT-D and CUHK sketch databases, respectively. Moreover, the eyelids segmentation from proposed method gives approximately 3.9783 standard deviation of the absolute mean error. Despite of this, there are some limitations to this proposed method. Firstly, the proposed method requires the sketch input image with the face region dimensions of at least $200 \times 200$. This is to avoid degraded performance of the iris and eyelids detection. Next, the minimum and maximum values for the iris of the sketch images need to be considered in order to acquire the best iris segmentation result. Also image binarization process in the preprocessing stage can be improved by considering other thresholding method. In addition to that, the proposed method will perform poorly on the eye image that have poor illumination or grayscale information. Dark image and rotated images are among the examples. The performance of the system is also affected by external factors such as the sketch image with occlusions (e.g. spectacle, eye region that is partially covered by hair) and image qualities (e.g. multilayer eyelid images, dark eye region). These factors causing the system to be less reliable. Therefore, we will look into these limitation in our future work.

\section{Acknowledgement}

This research work is fully supported by Universiti Sains Malaysia Research University Individual (RUI) Research Grant Scheme no. 1001/PELECT/814208.

\section{References}

[1] B. Klare, Z. Li, A. K. Jain, "Matching Forensic Sketches to Mug Shot Photos", IEEE Transactions on Pattern Analysis and Machine Intelligence, 33(3):639-646, 2011. doi: 10.1109/TPAMI.2010.180.

[2] X. Wang, X. Tang, "Face Photo-Sketch Synthesis and Recognition", IEEE Transactions on Pattern Analysis and Machine Intelligence, 31(11):1955-1967, 2009. doi: 10.1109/TPAMI.2008.222.

[3] R. U. Jr, N. D. V. Lobo, ”A Framework for Recognizing A Facial Image from A Police Sketch", Computer Vision and Pattern Recognition, 586-593, 1996. doi: 10.1109/CVPR.1996.517132.

[4] S. Zhang, X. Gao, N. Wang, J. Li, M. Zhang, "Face Sketch Synthesis via Sparse RepresentationBased Greedy Search", IEEE Transactions on Image Processing, 24(8):2466-2477, 2015. doi: 10.1109/TIP.2015.2422578.

[5] L. Chang, Y. Rozenholc, X. Deng, F. Duan, M. Zhou, "Face Sketch Synthesis using Non-Local Means and Patch-Based Seaming", International Conference on Image Processing(ICIP), 1508-1512, 2015. doi: 10.1109/ICIP.2015.7351052. 
[6] S. Buoncompagni, A. Franco, D. Maio, "Shape Features for Candidate Photo Selection in Sketch Recognition", International Conference on Pattern Recognition, 1728-1733, 2014. doi: 10.1109/ICPR.2014.304.

[7] S. Klum, H. Han, A. K. Jain, B. Klare, "Sketch Based Face Recognition: Forensic vs. Composite Sketches", International Conference on Biometrics, ICB2013, 1-8, 2013. doi: 10.1109/ICB.2013.6612993.

[8] B. F. Klare, A. K. Jain, "Heterogeneous Face Recognition using Kernel Prototype Similarities", IEEE Transactions on Pattern Analysis and Machine Intelligence, 35(6):1410-1422, 2013. doi: 10.1109/TPAMI.2012.229.

[9] S. Ouyang, T. Hospedales, Y. Z. Song, X. Li, "Cross-Modal Face Matching: Beyond Viewed Sketches", Lecture Notes in Computer Science, 9004:210-225, 2015. doi: 10.1007/978-3-319-16808-1_15.

[10] B. F. Klare, S. Klum, J. C. Klontz, E. Taborsky, T. Akgul, A. K. Jain, ”Suspect Identification Based on Descriptive Facial Attributes", IEEE/IAPR International Joint Conference on Biometrics, IJCB2014, 1-8, 2014. doi: 10.1109/BTAS.2014.6996255.

[11] C. Ma, X. Yang, C. Zhang, X. Ruan, M. H. Yuang, "Sketch Retrieval via Local Dense Stroke Features", Image and Vision Computing, 46:64-73, 2016. doi: 10.1016/j.imavis.2015.11.007.

[12] X. Tang, X. Wang, "Face Photo Recognition using Sketch", IEEE 2002 International Conference on Image Processing, 257-260, 2002. doi: 10.1109/ICIP.2002.1038008.

[13] X. Tang, X. Wang, "Face Sketch Recognition", IEEE Transactions on Circuits and Systems for Video Technology, 14(1):50-57, 2004. doi: 10.1109/TCSVT.2003.818353.

[14] H. Bhatt, S. Bharadwaj, R. Singh, M. Vatsa, "On Matching Sketches with Digital Face Images", IEEE Biometrics COmpendium, 1-7, 2010. doi: 10.1109/BTAS.2010.5634507.

[15] P. Viola, M. Jones, "Rapid Object Detection using a Boosted Cascade of Simple Features", Computer Vision and Pattern Recognition (CVPR), 1:511-518, 2001. doi: 10.1109/CVPR.2001.990517.

[16] D. Singh, R. Gupta, "Matching Facial Composite Sketches to Police Mug-Shot Images Based on Geometric Features", IOSR Journal of Computer Engineering (IOSR-JCE), 16(3):29-35, 2014.

[17] H. Yuen, J. Princen, J. Illingworth, J. Kittler, "Comparative Study of Hough Transform Methods for Circle Finding”, Image and Vision Computing, 8(1):71-77, 1990. doi: 10.1016/0262-8856(90)90059-E.

[18] T. J. Atherton, D. J. Kerbyson, "Size Invariant Circle Detection", Image and Vision Computing, 17(11):795-803, 1999. doi: 10.1016/S0262-8856(98)00160-7.

[19] A. Radman, N. Zainal, M. Ismail, "Efficient Iris Segmentation Based on Eyelid Detection", Journal of Engineering Science and Technology, 8(4):399-405, 2013.

[20] A. Radman, K. Jumari, N. Zainal, "Fast and Reliable Iris Segmentation Algorithm", IET Image Processing, 7(1):42-49, 2013. doi: 10.1049/iet-ipr.2012.0452.

[21] MATLAB, "MATLAB Mathematics: Polynomial Curve Fitting", 2016b, Natick, Massachusetts: The MathWorks Inc., 2016.

[22] H. Hofbauer, F. Alonso-Fernandez, P. Wild, J. Bigun, A. Uhl, "A Ground Truth for Iris Segmentation", International Conference on Pattern Recognition, 527-532, 2014. doi: 10.1109/ICPR.2014.101.

[23] Z. Zhao, A. Kumar, "An Accurate Iris Segmentation Framework under Relaxed Imaging Constraints using Total Variation Model", International Conference on Computer Vision, 3828-3836, 2015. doi: 10.1109/ICCV.2015.436. 
[24] P. C. Yuen, C. H. Man, "Human face image searching system using sketches" IEEE Transactions on Systems, Man, and Cybernetics Part A: Systems and Humans, 37(4):493504, 2007. doi: 10.1109/TSMCA.2007.897588.

[25] D. W. Hansen, Q. Ji, "In the eye of the beholder: a survey of models for eyes and gaze", IEEE transactions on pattern analysis and machine intelligence, 32(3):478500, 2010. doi: 10.1109/TPAMI.2009.30.

[26] G. C. Feng, P. C. Yuen, "Multi-cues eye detection on gray intensity image", Pattern Recognition, 34(5):10331046, 2001. doi: 10.1016/S0031-3203(00)00042-X.

[27] G. C. Feng, P. C. Yuen, "Variance projection function and its application to eye detection for human face recognition", Pattern Recognition Letters, 19:899906, 1998. doi: 10.1016/S0167-8655(98)00065-8.

[28] R. Stiefelhagen, A. Waibel, "A model-based gaze tracking system", Proceedings IEEE International Joint Symposia on Intelligence and Systems, 304310, 1996. doi: 10.1109/IJSIS.1996.565083.

[29] J. Huang, D. Ii, X. Shao, H. Wechsler, L. Martin, ”Pose Discriminiation and Eye Detection Using Support Vector Machines ( SVM )", Proceeding of NATOASI on Face Recognition: From Theory to Applications, 1998. doi: 10.1109/ICPR.1998.711102.

[30] F. Jiao, G. He, "Real-Time Eye Detection and Tracking under Various Light Conditions", Data Science Journal, 6:S636S640, 2007. doi: 10.1145/507072.507100.

[31] P. M. Hillman, J. M. Hannah, P. M. Grant, "Global fitting of a facial model to facial features for modelbased video coding", International Symposium on Image and Signal Processing and Analysis, ISPA, 1(I):359364, 2003. doi: 10.1109/ISPA.2003.1296923.

[32] I. Fasel, B. Fortenberry, J. Movellan, "A generative framework for real time object detection and classification", Computer Vision and Image Understanding, 98(1):182210, 2005. doi: 10.1016/j.cviu.2004.07.014.

[33] P. Kuo, J. Hannah, "An improved eye feature extraction algorithm based on deformable templates" Proceedings - International Conference on Image Processing, ICIP, 2:12061209, 2005. doi: 10.1109/ICIP.2005.1530278.

[34] D. W. Hansen, J. P. Hansen, M. Nielsen, A. S. Johansen, M. B. Stegmann, ”Eye typing using Markov and active appearance models" Proceedings of IEEE Workshop on Applications of Computer Vision, 132136, 2002. doi: 10.1109/ACV.2002.1182170. 